\title{
Efficacy of olanzapine long-acting injection in patients with acutely exacerbated schizophrenia: an insight from effect size comparison with historical oral data
}

\author{
Holland C Detke ${ }^{1 *}$, Fangyi Zhao ${ }^{1}$ and Michael M Witte ${ }^{2}$
}

\begin{abstract}
Background: To treat acute schizophrenia, a long-acting injectable antipsychotic needs a rapid onset of action and therapeutic profile similar to that of oral agents. The present post-hoc analyses compared results from a randomized, double-blind, placebo-controlled trial of olanzapine long-acting injection (LAl) for acute schizophrenia with those observed in similarly designed trials of oral olanzapine.

Methods: Six-week results from the olanzapine LAl study $(N=404)$ were compared with those of 3 oral studies (study 1: olanzapine vs. haloperidol vs. placebo [N=335]; study 2: olanzapine vs. haloperidol vs. low-dose olanzapine [ $\mathrm{N}=431]$; study 3: olanzapine vs. placebo vs. low-dose olanzapine [ $\mathrm{N}=152]$ ). All patients had baseline Brief Psychiatric Rating Scale (BPRS) scores $\geq 24$ (0-6 scale). Six-week effect sizes were calculated. Efficacy onset, pharmacokinetics, discontinuations, weight gain, and extrapyramidal symptoms were also assessed.

Results: At 6 weeks, mean BPRS scores decreased by 14 to 15 points for olanzapine LAl (405 mg/4 weeks, 210 or $300 \mathrm{mg} / 2$ weeks), by 8 to 16 for oral olanzapine (10 2.5 or $15 \pm 2.5 \mathrm{mg} /$ day), and by 12 to 13 for haloperidol $(15 \pm 5 \mathrm{mg} /$ day). For those same dose groups, effect sizes vs. placebo for the BPRS were 0.7 to 0.8 for olanzapine LAI, 0.5 to 0.7 for oral olanzapine, and 0.6 for haloperidol. The first statistically significant separation from placebo on the BPRS occurred at 3 days for the olanzapine LAl groups and at 1 week for oral olanzapine and haloperidol $(15 \pm 5 \mathrm{mg} /$ day) in oral study 1 although as late as week 6 for the 10-mg/day olanzapine dose in oral study 3 . Olanzapine concentrations were similar across studies. Weight gain $\geq 7 \%$ of baseline occurred in up to $35 \%$ of olanzapine LAI and oral patients versus up to $12 \%$ of haloperidol and placebo patients. Extrapyramidal symptoms were lowest in the olanzapine LAl groups and significantly greater in the haloperidol groups. No post-injection delirium/sedation syndrome events occurred in the olanzapine LAI study.

Conclusions: Patients treated acutely with olanzapine LAI showed a similar pattern of improvement to that seen historically with oral olanzapine. With the exception of injection-related adverse events, the efficacy and tolerability profile of olanzapine LAl is similar to oral olanzapine.

Trial registration: ClinicalTrials.gov ID; URL: http//www.clinicaltrials.gov/: NCT00088478; ClinicalStudyResults.org ID; URL: http://www.clinicalstudyresults.org/: 917, 978, 982, and 5984.
\end{abstract}

Keywords: Olanzapine, Long-acting injection, Pamoate, Haloperidol, Effect size, Onset, Pharmacokinetics

\footnotetext{
* Correspondence: detkehc@lilly.com

${ }^{1}$ Eli Lilly and Company, Indianapolis, IN, USA

Full list of author information is available at the end of the article
} 


\section{Background}

In the treatment of patients with acute symptoms of schizophrenia, second generation oral antipsychotics have been the treatment of choice, and one of the standards of care is oral olanzapine [1]. In the United States, the use of long-acting formulations have been reserved for patients who have difficulty complying with oral regimens during maintenance treatment [2-4], and little information is available about their use for patients with acute symptoms of schizophrenia [5]. Given the high frequency of insufficient medication adherence among patients with schizophrenia [6-8], it could be clinically advantageous to be able to initiate a long-acting depot antipsychotic during an acute exacerbation. To benefit patients with acute symptoms, a long-acting antipsychotic formulation needs a rapid onset of action, combined with a therapeutic profile similar to that of second generation oral agents.

Olanzapine long-acting injection (LAI) has demonstrated efficacy in acutely ill patients [9] and has demonstrated similarity to oral olanzapine in terms of maintenance of effect and safety during longer-term treatment [10]; several recent reviews are available [11-13]. However, no direct comparisons of oral and LAI olanzapine have been done within the acute phase of treatment. In order to address this question, a post-hoc analysis of effect sizes was conducted to compare efficacy results from the acute trial of olanzapine LAI [9] with historical data from 3 similarly designed trials of oral olanzapine for the acute treatment of schizophrenia [14-16]. The 3 oral studies were randomized, controlled trials using either placebo $[14,16]$ or a nontherapeutic dose of oral olanzapine $(1 \mathrm{mg} /$ day $)$ as the reference group $[15,16]$. In addition, oral haloperidol was used as an active comparator in 2 of these oral olanzapine trials $[14,15]$, thus potentially allowing for further clinical context for the olanzapine LAI findings. As in the acute olanzapine LAI trial, the oral trials required patients to be acutely symptomatic, and mean Brief Psychiatric Rating Scale (BPRS) scores (0-6 scale) were similar across all 4 studies, in the range of 37 to 43, indicating markedly ill patient populations. While not providing the degree of control possible in a direct head-to-head comparison, the use of effect sizes allows for useful comparisons of efficacy data across trials that employ similar designs and patient populations. In addition to evaluating effect sizes, we examined onset of efficacy, defined as the time of first statistical separation from placebo or a non-therapeutic dose of olanzapine. Finally, we also provide indirect comparison of key tolerability measures across these trials.

\section{Methods}

\section{Study criteria}

To be included in the analyses, studies had to be randomized, double-blind, fixed- or semi-fixed-dose, placebocontrolled and/or employing a low non-therapeutic reference dose as control group, at least 6 weeks in length, and conducted in acutely ill patients with schizophrenia. All were also previously submitted to the United States Food and Drug Administration as part of the submission for approval for the treatment of schizophrenia. The 4 studies meeting these criteria were olanzapine LAI study F1D-MC-HGJZ [9], oral study F1D-MCHGAD [14], oral study F1D-EW-E003 [15], and oral study F1D-MC-HGAP [16] (See Table 1). All patients were diagnosed with schizophrenia using then-current Diagnostic and Statistical Manual of Mental Disorders (DSM) criteria and had to meet minimum BPRS criteria (at least $\geq 24$ ) to ensure that patients were acutely ill at study entry. Three of the studies compared the respective formulation of olanzapine (LAI or oral) with placebo $[9,14,16]$; two of the studies employed a non-therapeutic reference dose of oral olanzapine (1 mg/day) $[15,16]$. In all 4 studies, patients were required to be inpatients for at least the first 2 weeks of the study and could potentially become outpatients thereafter if clinically appropriate. The olanzapine LAI study excluded patients who were known to be treatment-resistant to olanzapine but did not exclude other treatment-resistant patients. Oral studies 1 and 2 excluded patients who were known to be clear non-responders to neuroleptic treatment. Oral study 3 excluded patients who had failed to show minimal clinical response to sufficient doses of at least 3 different neuroleptics from 3 different chemical classes or to a dose of $400 \mathrm{mg} / \mathrm{d}$ of clozapine for at least 6 weeks.

The BPRS total scores and/or the Positive and Negative Syndrome Scale (PANSS) total scores were used to assess efficacy. Pharmacokinetic sampling was conducted in all patients at the end of the visit, just prior to the next dose for oral patients or just prior to the next injection for LAI patients when at an injection visit. LAI patients also had assessments taken periodically between injections. Heparinized plasma samples were analyzed for olanzapine using a validated high-performance liquid chromatography method at the same centralized laboratory across all 4 studies. For the LAI study, samples collected $>1$ day before or after the intended visit interval were excluded from analysis. Safety measures included mean change in weight from baseline to endpoint and the incidence of treatment-emergent weight gain $\geq 7 \%$ from baseline. Treatment-emergent Parkinsonism was defined as a Simpson-Angus [17] total baseline score $\leq 3$ and a post-baseline score $>3$ at anytime. Treatmentemergent akathisia was defined as a Barnes Akathisia Rating Scale [18] baseline global score $<2$ and a postbaseline score $\geq 2$ at anytime. Patients were allowed to take benzodiazapines for insomnia, anxiety, or agitation ( $\leq 2 \mathrm{mg} /$ day lorazepam equivalents in the olanzapine LAI study, and $\leq 10 \mathrm{mg} /$ day in the 3 oral studies). Anticholinergic use for extrapyramidal symptoms was permitted 
Table 1 A summary of the olanzapine long-acting injection and oral olanzapine studies analyzed

\begin{tabular}{|c|c|c|c|c|c|c|}
\hline Study & Dates Conducted & Primary Objective & Design & $\begin{array}{l}\text { Treatments, Doses, } \\
\text { and Regimen }\end{array}$ & $N$ & $\begin{array}{l}\text { Diagnosis and Inclusion } \\
\text { Criteria }\end{array}$ \\
\hline \multirow[t]{5}{*}{ OLZ LAI [9] } & $\begin{array}{l}\text { Jun } 2004- \\
\text { Apr } 2005\end{array}$ & $\begin{array}{l}\text { Efficacy vs. placebo } \\
\text { (PANSS Total) }\end{array}$ & 2 - 7 day washout & OLZ LAI & 404 & DSM-IV schizophrenia \\
\hline & & & 8 weeks & $405 \mathrm{mg} / 4$ weeks & & BPRS score $\geq 30$ ( 0 - 6 scale) \\
\hline & & & Randomized, & $210 \mathrm{mg} / 2$ weeks & & \\
\hline & & & double-blind & 300 mg/2 weeks & & \\
\hline & & & & IM placebo/2 weeks & & \\
\hline \multirow[t]{6}{*}{$\begin{array}{l}\text { Oral OLZ, Study } 1 \\
{[14]}\end{array}$} & $\begin{array}{l}\text { Oct } 1991- \\
\text { Nov } 1996\end{array}$ & $\begin{array}{l}\text { Efficacy vs. placebo and } \\
\text { HAL (BPRS) }\end{array}$ & $\begin{array}{l}4-7 \text { day placebo } \\
\text { lead-in }\end{array}$ & OLZ & 335 & $\begin{array}{l}\text { DSM-III-R schizophrenia with } \\
\text { acute exacerbation }\end{array}$ \\
\hline & & & & $5 \pm 2.5 \mathrm{mg} /$ day & & \\
\hline & & & 6 weeks & $10 \pm 2.5 \mathrm{mg} /$ day & & BPRS score $\geq 24$ ( 0 - 6 scale) \\
\hline & & & Randomized, & $15 \pm 2.5 \mathrm{mg} /$ day & & \\
\hline & & & double-blind & HAL: $15 \pm 5$ mg/day & & \\
\hline & & & & Placebo & & \\
\hline \multirow[t]{6}{*}{$\begin{array}{l}\text { Oral OLZ, Study } 2 \\
{[15]}\end{array}$} & $\begin{array}{l}\text { Nov } 1991- \\
\text { Feb } 1997\end{array}$ & Efficacy vs. HAL (BPRS) & $\begin{array}{l}4-7 \text { day placebo } \\
\text { lead-in }\end{array}$ & OLZ & 431 & $\begin{array}{l}\text { DSM-III-R schizophrenia } \\
\text { with acute exacerbation }\end{array}$ \\
\hline & & & & $5 \pm 2.5 \mathrm{mg} /$ day & & \\
\hline & & & 6 weeks & $10 \pm 2.5 \mathrm{mg} /$ day & & BPRS score $\geq 24$ ( 0 - 6 scale) \\
\hline & & & Randomized, & $15 \pm 2.5 \mathrm{mg} /$ day & & CGI-S score $\geq 4$ \\
\hline & & & double-blind & 1 mg/day & & \\
\hline & & & & HAL: $15 \pm 5$ mg/day & & \\
\hline \multirow[t]{4}{*}{$\begin{array}{l}\text { Oral OLZ, Study } 3 \\
{[16]}\end{array}$} & $\begin{array}{l}\text { Jul } 1993- \\
\text { Jan } 1997\end{array}$ & Efficacy vs. Placebo (BPRS) & $\begin{array}{l}4-9 \text { day placebo } \\
\text { lead-in }\end{array}$ & OLZ & 152 & $\begin{array}{l}\text { DSM-III-R schizophrenia with } \\
\text { acute exacerbation }\end{array}$ \\
\hline & & & & 1 mg/day & & \\
\hline & & & 6 weeks & 10 mg/day & & BPRS score $\geq 24$ ( 0 - 6 scale) \\
\hline & & & $\begin{array}{l}\text { Randomized, } \\
\text { double-blind }\end{array}$ & Placebo & & \\
\hline
\end{tabular}

Abbreviations: BPRS: Brief Psychiatric Rating Scale; CGI-S: Clinical Global Impressions - Severity; DSM: Diagnostic and Statistical Manual (versions III-R: revised; and IV); OLZ: olanzapine; HAL: haloperidol; OLZ LAl: olanzapine long-acting injection; PANSS: Positive and Negative Syndrome Scale

( $\leq 6 \mathrm{mg} /$ day biperiden equivalents in all 4 studies), but prophylatic use was prohibited. No oral antipsychotic supplementation was allowed in the olanzapine LAI study. All 4 studies were conducted in accordance with the ethical principles of the Declaration of Helsinki. Protocols were conducted consistent with good clinical practices and all applicable laws and regulations in each region. Ethical Review Boards approved each protocol before investigators initiated the trials. All patients or their legal representative signed an informed consent before participation in the trial. Detailed information about each study has been published elsewhere [9,14-16].

\section{Statistical analyses}

The data in each of the 4 studies were analyzed on an intent-to-treat basis, and analysis results from each study are presented separately here. Standard baseline characteristics were summarized for all randomized patients. Mean changes from baseline to endpoint were analyzed using the last-observation-carried-forward approach.
Differences between groups in continuous data within each study were assessed using analysis of variance, with treatment, investigator (or country), and/or treatmentby-investigator (or treatment-by-country) interaction as fixed factors. Differences between groups in categorical data were assessed with Fisher's exact test or Pearson's chi-square test. All comparisons were conducted at a two-sided alpha level of 0.05 without adjustment for multiplicity. Onset of efficacy was defined as the first time point at which a treatment group achieved statistical superiority to the reference group (placebo or low non-therapeutic dose) followed by statistical superiority at all subsequent visits.

Effect size for each treatment group was calculated against placebo or the non-therapeutic dose of oral olanzapine ( $1 \mathrm{mg} /$ day) as the reference group. Effect size was calculated as the difference of mean changes in efficacy measures (BPRS and PANSS total scores) of the treatment group and the reference group (placebo or oral olanzapine $1 \mathrm{mg} /$ day), divided by their pooled standard 
deviations. Because the oral olanzapine studies were only 6 weeks in length while the olanzapine LAI study was 8 weeks in length, only the first 6 weeks of data from the olanzapine LAI study were used for the betweenstudy efficacy comparison; however, pharmacokinetic and safety comparisons were not adjusted for time in treatment.

\section{Results}

\section{Baseline symptom severity}

Patient demographics and baseline symptom severity were similar across the 4 studies (Table 2). Patients were mostly male (64-88\%) and Caucasian (56-86\%), with mean ages of 36 to 41 years.

\section{Efficacy assessments}

Weekly reduction in symptoms assessed by the BPRS in each of the 4 studies over 6 weeks are shown in Figure 1. Three of the 4 studies also evaluated symptom changes with the PANSS (olanzapine LAI study, oral study 2, and oral study 3), and those results are shown in Additional file 1: Figure S1.

\section{Effect size}

Table 3 shows the effect sizes for the BPRS and PANSS total assessments for each active treatment group in all 4 studies. Most importantly, the placebo-controlled effect sizes for treatment with olanzapine LAI were comparable to the effect sizes for treatment with oral olanzapine seen in each of the 3 studies. Effect sizes in oral olanzapine study 2 were lower, but this is due to the fact that the 1-mg/day oral olanzapine treatment group was used as the reference group instead of placebo.

Table 2 Patient demographics and baseline illness characteristics

\begin{tabular}{|c|c|c|c|c|}
\hline Characteristic & $\begin{array}{c}\text { OLZ LAI } \\
\text { Study } \\
(\mathrm{N}=404)\end{array}$ & $\begin{array}{c}\text { Oral } \\
\text { Study } 1 \\
(\mathrm{~N}=335)\end{array}$ & $\begin{array}{c}\text { Oral } \\
\text { Study } 2 \\
(\mathrm{~N}=431)\end{array}$ & $\begin{array}{c}\text { Oral } \\
\text { Study } 3 \\
(N=152)\end{array}$ \\
\hline Male, n (\%) & $285(70.5)$ & $294(87.8)$ & $275(63.8)$ & $110(72.4)$ \\
\hline Caucasian, n (\%) & $226(55.9)$ & $230(68.7)$ & $372(86.3)$ & $104(68.4)$ \\
\hline Mean Age, years (SD) & $40.8(11.2)$ & $36.0(9.4)$ & $35.5(10.7)$ & $37.6(9.2)$ \\
\hline $\begin{array}{l}\text { Mean Age of Disease } \\
\text { Onset, years (SD) }\end{array}$ & $23.4(8.2)$ & $22.0(5.8)$ & $24.1(7.7)$ & $21.7(5.7)$ \\
\hline $\begin{array}{l}\text { Median Length of } \\
\text { Current Episode, days }\end{array}$ & 39 & 30 & 32 & 40 \\
\hline $\begin{array}{l}\text { Mean PANSS Total } \\
\text { Score (SD) }\end{array}$ & $101.0(15.6)$ & - & $103.3(18.4)$ & $98.2(17.7)$ \\
\hline Mean BPRS Total (SD) & $40.9(8.9)$ & $41.5(11.0)$ & $40.7(10.6)$ & $38.0(9.0)$ \\
\hline
\end{tabular}

Abbreviations: BPRS: Brief Psychiatric Rating Scale; OLZ LAl: olanzapine longacting injection; PANSS: Positive and Negative Syndrome Scale; SD: standard deviation

\section{Onset of efficacy}

Onset of efficacy was demonstrated by all 3 doses of olanzapine LAI vs. placebo starting at day $3(p<.05)$ on the BPRS. On the PANSS, improvement vs. placebo began at day 3 for two of the olanzapine LAI dose groups (405 mg/ 4 weeks and $300 \mathrm{mg} / 2$ weeks; $p<.05$ ) and at week 1 for the other dose group $(210 \mathrm{mg} / 2$ weeks; $p<.01)$. In oral study 1 , the medium and high olanzapine dose groups (10 \pm 2.5 and $15 \pm 2.5 \mathrm{mg} /$ day) and the haloperidol group (15 $\pm 5 \mathrm{mg} /$ day) all demonstrated onset of efficacy compared with placebo starting at week $1(p<.05)$ on the BPRS; the low dose olanzapine group $(5 \pm 2.5 \mathrm{mg} /$ day $)$ separated from placebo only at week 2 of treatment $(p<.05)$. In oral study 2 , although a similar pattern of symptom reduction was seen in the active groups, no groups separated statistically from the very low reference dose comparator (1 mg/day olanzapine) at any time. In oral study 3 , onset of efficacy for the 10-mg/day olanzapine group began at the 6 -week endpoint vs. placebo on the BPRS $(p<.05)$ but began starting at week 2 on the PANSS $(p<.05)$.

\section{Olanzapine plasma concentrations}

The expected therapeutic range for olanzapine plasma concentrations for within-label doses of olanzapine (oral or LAI) has been reported as approximately 5 to $73 \mathrm{ng} /$ $\mathrm{mL}$, representing the $10^{\text {th }}$ percentile for the lowest withinlabel dose and the $90^{\text {th }}$ percentile for the highest within label dose [20]. In the LAI study, mean concentrations $(\mathrm{ng} / \mathrm{mL})$ were in the therapeutic range as early as day 3, with means of $9.1(\mathrm{SD}=8.6), 17.2(\mathrm{SD}=14.7)$, and 15.6 $(\mathrm{SD}=13.3)$ for the $210-\mathrm{mg} / 2$ week, $405-\mathrm{mg} / 4$ week, and $300-\mathrm{mg} / 2$ week groups, respectively. Table 4 presents olanzapine plasma concentrations across all studies at study endpoint. Although study endpoint for the olanzapine LAI study was at 8 weeks (vs. 6 weeks for the oral studies), endpoint results represent the best comparison as these indicate trough levels for each of the formulations and dosage regimens, including the 4-week LAI dosage which would otherwise not be at trough level at 6 weeks.

\section{Weight gain}

The safety results from the olanzapine LAI study show similar patterns of weight gain for patients in the 3 olanzapine LAI treatment groups as that reported for the therapeutic treatment groups in oral olanzapine studies 1,2 , and 3. At study endpoint, mean weight increased by $2.8-3.9 \mathrm{~kg}$ for olanzapine LAI ( $p \leq .001$ vs. placebo for all LAI doses), $1.7-3.6 \mathrm{~kg}$ for oral olanzapine (study $1: p<.05$ vs. placebo for all oral olanzapine doses and $p<.05$ vs. haloperidol for the medium and high olanzapine doses; study 2: $p<.001$ vs. $1 \mathrm{mg}$ oral and $p<.01$ vs. haloperidol for the medium and high olanzapine doses; study 3: $p<.05$ vs. $1 \mathrm{mg}$ and placebo), and $-0.4-0.9 \mathrm{~kg}$ for haloperidol (not significant vs. placebo or $1 \mathrm{mg} /$ day olanzapine). Weight gain $\geq 7 \%$ of 


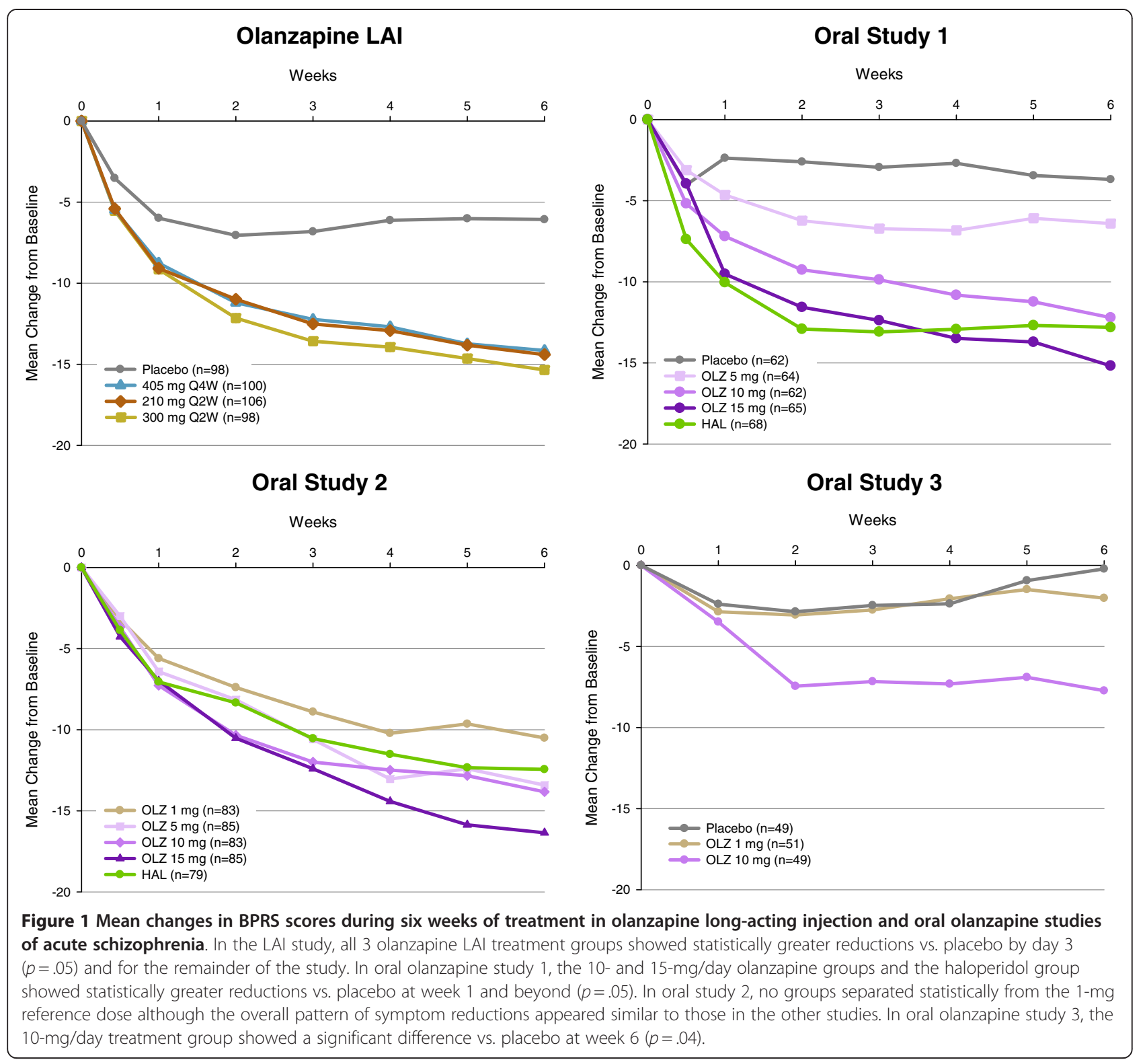

baseline was statistically significantly greater in the olanzapine groups, but comparable between the studies for olanzapine-treated patients: olanzapine LAI vs. placebo $(24 \%-35 \%$ vs. $12 \%, p$ 's $<.05)$; study 1 oral olanzapine $(28 \%-34 \%)$ vs. placebo $(3 \%$, p's $<.001)$ and haloperidol $(12 \%, p<.05)$; study 2 oral olanzapine $(19 \%-35 \%)$ vs. $1 \mathrm{mg}$ oral $(13 \%, p$ 's $<.05)$ and haloperidol $(4 \%, p<.05)$; and study 3 oral olanzapine $(20 \%)$ vs. $1 \mathrm{mg}$ oral $(6 \%, p<.05)$ and placebo $(2 \%, p<.01)$.

\section{Extrapyramidal symptoms}

Overall incidence of treatment-emergent extrapyramidal symptoms was lower across all groups in the LAI study than in the older oral studies, including patients in the placebo and non-therapeutic control groups, but was markedly lower for the LAI and oral olanzapine groups than the haloperidol groups. Incidence of Parkinsonism in the olanzapine LAI study showed no statistically significant difference between drug and placebo (2\%-6\% olanzapine LAI vs. $6 \%$ placebo), nor was there a significant difference between therapeutic oral olanzapine doses and placebo (study 1: $12 \%-14 \%$ oral olanzapine vs. $15 \%$ placebo; study 3 : $7 \%$ oral olanzapine vs. $8 \%$ placebo). However, incidence of Parkinsonism in the haloperidol treatment groups was significantly higher than in the therapeutic oral olanzapine groups in both oral study 1 ( $42 \%$ vs. $12 \%-14 \%$, $p$ 's $\leq .001$ ) and study 2 (53\% vs. $14 \%-19 \%, p$ 's $<.001)$. Akathisia showed a similar pattern, with no significant differences between olanzapine LAI and placebo (1\%-6\% olanzapine LAI vs. $6 \%$ placebo) or therapeutic oral olanzapine and placebo 
Table 3 Effect sizes for olanzapine oral or long-acting injection vs. placebo or non-therapeutic olanzapine (1 mg)

\begin{tabular}{|c|c|c|c|c|c|c|}
\hline Study and Treatment & $\mathrm{N}$ & $\begin{array}{l}\text { Mean Modal Dose/ } \\
\text { Corresponding } \\
\text { Daily Dose } \\
\text { (mg/day) }\end{array}$ & $\begin{array}{l}\text { PANSS Total } \\
\text { LS Mean } \\
\text { Change }\end{array}$ & $\begin{array}{l}\text { PANSS Total } \\
\text { Effect Size }^{a} \\
\text { (category) }^{b}\end{array}$ & $\begin{array}{l}\text { BPRS Total } \\
\text { LS Mean } \\
\text { Change }\end{array}$ & $\begin{array}{l}\text { BPRS Total } \\
\text { Effect Size } \\
\text { (category) }^{\mathrm{b}}\end{array}$ \\
\hline \multicolumn{7}{|l|}{ OLZ LAI Study } \\
\hline 405 mg/4 weeks olanzapine LAl & 100 & 14.5 & -22.5 & .75 (moderate) & -14.2 & .71 (moderate) \\
\hline 210 mg/2 weeks olanzapine LAl & 106 & 15.0 & -22.8 & .80 (large) & -14.4 & .77 (moderate) \\
\hline 300 mg/2 weeks olanzapine LAl & 100 & 21.4 & -24.8 & .88 (large) & -15.4 & .82 (large) \\
\hline vs. Placebo & 98 & 0.0 & -8.7 & - & -6.1 & - \\
\hline \multicolumn{7}{|l|}{ Oral Study 1} \\
\hline $5 \pm 2.5 \mathrm{mg} /$ day olanzapine & 65 & 6.6 & - & - & -6.4 & .17 (small) \\
\hline $10 \pm 2.5 \mathrm{mg} /$ day olanzapine & 64 & 11.6 & - & - & -12.2 & .50 (moderate) \\
\hline $15 \pm 2.5 \mathrm{mg} /$ day olanzapine & 69 & 16.3 & - & - & -15.2 & .67 (moderate) \\
\hline $15 \pm 5.0 \mathrm{mg} /$ day haloperidol & 69 & 16.4 & - & - & -12.8 & .57 (moderate) \\
\hline vs. Placebo & 68 & 0.0 & - & - & -3.7 & - \\
\hline \multicolumn{7}{|l|}{ Oral Study 2} \\
\hline $5 \pm 2.5 \mathrm{mg} /$ day olanzapine & 87 & 6.7 & -21.4 & .17 (small) & -13.4 & .19 (small) \\
\hline $10 \pm 2.5 \mathrm{mg} /$ day olanzapine & 86 & 11.3 & -22.7 & .20 (small) & -13.8 & .19 (small) \\
\hline $15 \pm 2.5 \mathrm{mg} /$ day olanzapine & 89 & 16.4 & -26.7 & .37 (moderate) & -16.4 & .38 (moderate) \\
\hline $15 \pm 5.0 \mathrm{mg} /$ day haloperidol & 81 & 17.6 & -20.0 & .12 (small) & -12.4 & .12 (small) \\
\hline vs. $1.0 \mathrm{mg} /$ day olanzapine & 88 & 0.0 & -16.8 & - & -10.5 & - \\
\hline \multicolumn{7}{|l|}{ Oral Study 3} \\
\hline 10 mg/day olanzapine (vs. placebo) & 50 & 10.0 & -12.3 & .71 (moderate) & -7.7 & 60 (moderate) \\
\hline 10 mg/day olanzapine (vs. 1 mg) & 50 & 10.0 & -12.3 & .48 (moderate) & -7.7 & .45 (moderate) \\
\hline 1 mg/day olanzapine (vs. placebo) & 52 & 1.0 & -1.9 & .22 (moderate) & -2.0 & .14 (small) \\
\hline Placebo & 50 & 0.0 & 2.8 & - & -0.2 & - \\
\hline
\end{tabular}

${ }^{a}$ All effect sizes were calculated from baseline-to-endpoint LOCF mean change scores at 6 weeks of treatment (OLZ LAl study was 8 weeks, whereas the 3 oral studies were only 6 weeks)

${ }^{\mathrm{b}}$ Effect sizes were categorized using Cohen's general effect size thresholds [19] Abbreviations: BPRS: Brief Psychiatric Rating Scale; LOCF: last observation carried forward; LS: least squares; OLZ LAl: olanzapine long-acting injection; PANSS: Positive and Negative Syndrome Scale

(study 1: 16\%-27\% oral olanzapine vs. 23\% placebo; study 3: $13 \%$ oral olanzapine vs. $13 \%$ placebo). However, incidence of akathisia in the haloperidol groups was significantly higher than in the therapeutic oral olanzapine groups in both oral study 1 (46\% vs. $16 \%-27 \%$, p's $<.05$ ) and study 2 (34\% vs. $9 \%-12 \%$, $p$ 's $<.01)$.

\section{Discontinuation rates}

In Table 5, results from all therapeutically dosed olanzapine treatment groups were combined within each of the 4 studies to provide an overall rate of discontinuation compared with the placebo group and the nontherapeutic dose of oral olanzapine ( $1 \mathrm{mg} /$ day) used in oral studies 2 and 3 . The rate of discontinuations from any cause was lower in the olanzapine LAI study (combined dosages of olanzapine LAI) compared with placebo and was also lower than the rates seen in oral olanzapine studies 1 and 2 (combined dosages of oral olanzapine, haloperidol, placebo, and non-therapeutic dose of oral olanzapine) and in oral olanzapine study 3 (oral olanzapine $10 \mathrm{mg} /$ day, non-therapeutic dose of oral olanzapine, and placebo). The rate of discontinuation due to adverse events among patients treated with olanzapine LAI was comparable to patients in the placebo group. Differences in overall discontinuation rate across studies appeared to be driven primarily by higher rates of discontinuation due to lack of efficacy in the oral olanzapine studies 1 and 3 relative to the olanzapine LAI study. The incidence of discontinuation due to an adverse event for oral olanzapine in study 2 (11\%) was more than twice that of olanzapine LAI (4\%), but no statistical comparisons were made between the studies regarding discontinuation rates.

\section{Discussion}

This cross-study, post-hoc analysis indicates that patients with acutely exacerbated schizophrenia treated with olanzapine LAI dosages of $405 \mathrm{mg} / 4$ weeks, 
Table 4 Olanzapine plasma concentrations at study endpoint for the olanzapine long-acting injection and olanzapine oral studies

\begin{tabular}{|c|c|c|c|c|c|}
\hline Study and Treatment & $\mathbf{n}$ & $\begin{array}{l}\text { Mean at } \\
\text { endpoint } \\
\text { (ng/mL) }\end{array}$ & SD & Minimum & Maximum \\
\hline
\end{tabular}

\section{OLZ LAI Study}

$405 \mathrm{mg} / 4$ weeks

olanzapine LAI

$210 \mathrm{mg} / 2$ weeks

olanzapine LAI

$300 \mathrm{mg} / 2$ weeks olanzapine LAI

$\begin{array}{rrrrr}39 & 13.4 & 7.8 & 5.9 & 56.0 \\ 55 & 18.3 & 9.4 & 5.5 & 44.7 \\ 56 & 24.3 & 11.6 & 9.3 & 87.6\end{array}$

\section{Oral Study 1}

$5 \pm 2.5 \mathrm{mg} /$ day
olanzapine
$10 \pm 2.5 \mathrm{mg} /$ day
olanzapine
$15 \pm 2.5 \mathrm{mg} /$ day
olanzapine

$\begin{array}{rrrrr}63 & 10.3 & 8.0 & 0.4 & 36.9 \\ 62 & 18.4 & 12.1 & 0.4 & 63.2 \\ 63 & 30.6 & 22.7 & 0.5 & 108.1\end{array}$

\section{Oral Study 2}

\begin{tabular}{lccccc}
$\begin{array}{l}1 \mathrm{mg} / \text { day } \\
\text { olanzapine }\end{array}$ & 75 & 1.7 & 1.9 & 0.3 & 16.0 \\
$\begin{array}{l}5 \pm 2.5 \mathrm{mg} / \text { day } \\
\text { olanzapine }\end{array}$ & 78 & 9.1 & 5.9 & 0.5 & 37.0 \\
$\begin{array}{l}10 \pm 2.5 \mathrm{mg} / \text { day } \\
\text { olanzapine }\end{array}$ & 76 & 16.8 & 11.5 & 0.3 & 64.3 \\
$\begin{array}{l}15 \pm 2.5 \mathrm{mg} / \text { day } \\
\text { olanzapine }\end{array}$ & 75 & 26.9 & 19.2 & 1.2 & 99.8 \\
$\begin{array}{l}\text { Oral Study 3 } \\
\begin{array}{l}1 \mathrm{mg} / \text { day } \\
\text { olanzapine }\end{array}\end{array}$ & 51 & 1.5 & 0.8 & 0.0 & 3.2 \\
$\begin{array}{l}10 \mathrm{mg} / \text { day } \\
\text { Olanzapine }\end{array}$ & 48 & 20.7 & 17.1 & 0.3 & 93.0 \\
\hline
\end{tabular}

Abbreviations: $n$ : number of patients with evaluable samples; OLZ LAI: olanzapine long-acting injection; SD: standard deviation
$210 \mathrm{mg} / 2$ weeks, and $300 \mathrm{mg} / 2$ weeks had a similar magnitude of symptom reduction as patients treated with $10 \pm 2.5 \mathrm{mg} /$ day and $15 \pm 2.5 \mathrm{mg} /$ day of oral olanzapine and $15 \pm 5 \mathrm{mg} /$ day of oral haloperidol during 6 weeks of acute treatment. Using historical clinical data for oral olanzapine provided a helpful framework for understanding the relative efficacy and tolerability of the long-acting formulation of olanzapine.

\section{Effect sizes}

Importantly, the 4 studies presented in this analysis were not conducted concurrently, and cross-study comparisons should be interpreted with caution. Therefore, effect size calculations were performed to allow a standardized comparison among the 4 studies. In general, an effect size above 0.5 standard deviations is considered "large" [19]. Comparison of effect sizes among the 4 studies suggests that the magnitude of symptom reduction seen with the 3 doses of olanzapine LAI was "large" and generally similar to that of approximately 10 to $15 \mathrm{mg} /$ day oral olanzapine or $15 \mathrm{mg} /$ day oral haloperidol. The evaluation of assessment scores and relative effect sizes in this analysis demonstrate that despite clinical and research changes over the years, the response to treatment with olanzapine LAI as measured against placebo was comparable to results from earlier oral olanzapine clinical trials against placebo.

\section{Onset of efficacy}

The post-hoc exploration of early onset of action based on mean change in symptoms measured by the BPRS or PANSS total scales in each study suggests that speed of onset with olanzapine LAI may be at least as rapid as that of therapeutic doses of oral olanzapine and oral haloperidol. Patients in all 3 treatment groups in the olanzapine LAI study achieved statistically significant reduction in symptoms within 1 week of receiving their first injection, which was comparable to or better than results observed for oral olanzapine in studies 1,2 , and 3 . Of note in the

Table 5 Discontinuation rates for the olanzapine long-acting injection and olanzapine oral studies

\begin{tabular}{|c|c|c|c|c|c|c|c|c|c|c|c|}
\hline & \multicolumn{2}{|c|}{ OLZ LAI (N=404) } & \multicolumn{3}{|c|}{ Oral Study $1(\mathrm{~N}=335)$} & \multicolumn{3}{|c|}{ Oral Study $2(\mathrm{~N}=431)$} & \multicolumn{3}{|c|}{ Oral Study $3(\mathrm{~N}=152)$} \\
\hline & $\begin{array}{c}\text { PLC } \\
(n=98)\end{array}$ & $\begin{array}{c}\text { OLZ LAI } \\
(n=306)^{a}\end{array}$ & $\begin{array}{c}\text { PLC } \\
(n=68)\end{array}$ & $\begin{array}{c}\text { HAL } \\
(n=68)\end{array}$ & $\begin{array}{c}\text { OLZ } \\
(n=198)^{a}\end{array}$ & $\begin{array}{c}1 \mathrm{mg} \\
(\mathrm{n}=88)\end{array}$ & $\begin{array}{c}\text { HAL } \\
(n=81)\end{array}$ & $\begin{array}{c}\text { OLZ } \\
(n=262)^{a}\end{array}$ & $\begin{array}{c}\text { PLC } \\
(n=50)\end{array}$ & $\begin{array}{c}1 \mathrm{mg} \\
(n=52)\end{array}$ & $\begin{array}{c}\text { OLZ } \\
(n=50)\end{array}$ \\
\hline \multicolumn{12}{|l|}{$\begin{array}{l}\text { Discontinued } \\
(\%)\end{array}$} \\
\hline All Cause ${ }^{b}$ & 42.9 & 31.0 & 67.6 & 56.5 & 56.1 & 44.3 & 46.9 & 37.8 & 80.0 & 76.9 & 62.0 \\
\hline Lack of Efficacy & 24.5 & 11.4 & 47.1 & 27.5 & 32.3 & 18.2 & 19.8 & 14.1 & 74.0 & 61.5 & 56.0 \\
\hline Patient Decision & 9.2 & 11.8 & 2.9 & 10.1 & 10.6 & 9.1 & 7.4 & 7.3 & 5.8 & 2.0 & 2.0 \\
\hline Adverse Event & 5.1 & 4.2 & 10.3 & 8.7 & 5.1 & 11.4 & 14.8 & 10.7 & 0.0 & 9.6 & 4.0 \\
\hline $\begin{array}{l}\text { Lost at Follow- } \\
\text { up }\end{array}$ & 1.0 & 0.7 & 1.5 & 7.2 & 3.0 & 1.1 & 2.5 & 1.5 & 4.0 & 0.0 & 0.0 \\
\hline
\end{tabular}

a Therapeutic dose groups were pooled

${ }^{b}$ All-cause percentages also include discontinuations for reasons other than those shown

Abbreviations: $1 \mathrm{mg}$ : $1 \mathrm{mg} /$ day non-therapeutic dose of olanzapine; HAL: haloperidol; OLZ: olanzapine; LAl: long-acting injection; PLC: placebo 
olanzapine LAI study, the clinical trial protocol did not allow supplementation with any oral antipsychotic medication, including oral olanzapine. The ability to begin to realize improvement of acute symptoms following the first injection, without the need for additional oral antipsychotic medication for symptom control during the initial weeks or months of treatment, may simplify the treatment plans for patients with acute symptoms.

\section{Olanzapine plasma concentrations}

Pharmacokinetic evaluation indicated that olanzapine plasma concentrations were in the therapeutic range as early as day 3 for the LAI study. Moreover, endpoint concentrations were generally similar across the 4 studies. These findings are consistent with the clinical observations. Interestingly, while all LAI patients had endpoint concentrations within the therapeutic range, some oral patients had concentrations below that range, suggesting that some oral patients may have failed to take their dose.

\section{Safety and tolerability}

Weight gain is very commonly reported during treatment with some atypical antipsychotics such as olanzapine. In this analysis weight gain was most similar between olanzapine LAI and oral olanzapine in oral study 1 , with patients gaining a mean of 3.5 to $4 \mathrm{~kg}$ on the higher doses of olanzapine. Patients treated with olanzapine LAI or therapeutic doses of oral olanzapine gained significantly more weight than did those treated with haloperidol or placebo. A significantly greater percentage of patients treated with olanzapine LAI or therapeutic doses of oral olanzapine gained clinically significant amounts of weight than did those treated with haloperidol or placebo. Although these were all short-term studies, it is important to note that changes in metabolic parameters have also been reported during longterm treatment with olanzapine $[21,22]$. Therefore, the potential consequences of weight gain should be considered prior to starting olanzapine treatment. Patients receiving olanzapine should have their weight monitored regularly.

With respect to the development of extrapyramidal symptoms, a significantly greater percentage of patients treated with haloperidol experienced treatment-emergent Parkinsonism or akathisia compared with those treated with olanzapine LAI, oral olanzapine, or placebo. The observed differences between the olanzapine LAI study and the oral studies may be due in part to historical context. At the time that the oral studies were conducted, patients were more likely to have been treated with typical antipsychotics previously, which may have influenced rates of extrapyramidal symptoms in the study despite the washout period. Also, rater expectations regarding extrapyramidal symptoms may have been different at that time, and inclusion of a haloperidol arm may have also biased rater expectations regarding extrapyramidal symptoms.

\section{Post-injection delirium/sedation syndrome (PDSS)}

Although no cases of PDSS occurred during the acute olanzapine LAI study, it is important to note that this is a risk for this depot formulation. During other olanzapine LAI clinical trials, adverse events related to delirium and/ or excessive sedation (including coma) were identified in a small percentage of patients following injection. These events have been reported following $<0.1 \%$ of injections of olanzapine LAI in $<2 \%$ of patients [23]. Because of the risk of PDSS, safety precautions-including a post-injection observation period- must occur at the time of each injection. Clinicians should consider these factors when weighing the overall risks and benefits of olanzapine LAI for each patient.

\section{Study discontinuation rates}

The low discontinuation rates in the olanzapine LAI study are encouraging considering that discontinuation rates are high in clinical studies of treatments for schizophrenia, but it is important to recognize that discontinuation rates in actual clinical use remain to be determined. Patients who have difficulty adhering to medication schedules are usually not participants in controlled clinical studies [24-26]. Additionally, these studies employed both inpatient and outpatient treatment settings which may have influenced the overall study discontinuation rates. Nevertheless, the present findings suggest possible improvement in treatment persistence for patients who receive a long-acting injection.

\section{Limitations}

These findings are based on 4 separate studies, each analyzed separately, with a span of approximately 10 years between the oral olanzapine studies and the olanzapine LAI study. While effect size calculations help assess the magnitude of efficacy changes and comparison across baselines show similarities of symptoms, there are differences that must be considered when evaluating this information. Patient populations in these studies may have been different, particularly in their previous exposure to medications. Treatment paradigms at the time of the oral studies in the early 1990s were based on the use of first generation antipsychotic medications while current treatment includes the frequent use of second generation antipsychotic agents. It should also be noted that although the present analyses did not exclude any studies meeting our selection criteria, all studies were conducted by Lilly and did not include studies conducted by other sponsors.

It should also be noted that the present analyses are limited in their ability to provide comparisons of specific doses between oral and olanzapine LAI because of the differing length of time needed to reach steady state plasma concentrations for each olanzapine formulation. Olanzapine LAI does not reach steady-state concentrations of olanzapine 
for at least 3 months, while oral olanzapine should reach steady state levels within the first week of exposure. Therefore, the LAI results seen in the 6-week period examined in this work represent pre-steady state olanzapine levels, which are slightly lower than the levels which will ultimately be achieved over a longer period of time. For instance, while the $405 \mathrm{mg} / 4$ week dose may ultimately correspond to a $15 \mathrm{mg} /$ day oral dose, the short-term results presented here indicate that this dose may provide olanzapine concentrations more similar to a $10 \mathrm{mg} /$ day dose in the first 8 weeks of treatment. This is consistent with the pharmacokinetic and clinical findings from a previous study [27]. An additional consideration is that oral olanzapine studies 1 and 2 allowed semi-flexible dosing of the olanzapine target dose by $\pm 2.5 \mathrm{mg} /$ day while the olanzapine LAI study used strictly fixed doses and prohibited any oral antipsychotic supplementation.

An additional limitation is that only 2 of the 3 oral studies were placebo-controlled, and the 1-mg oral olanzapine reference dose did not perform like placebo with respect to safety measures and some small differences in treatment response. Therefore effect sizes vs. the 1-mg arm are not comparable with effect sizes vs. placebo.

\section{Conclusions}

Patients treated acutely with olanzapine LAI showed a similar pattern of improvement to that seen historically in 3 previous studies of oral olanzapine. Onset of action for the LAI formulation appeared as early as the first week after injection, with olanzapine plasma concentrations appearing similar across the LAI and oral studies. With the exception of injection-related adverse events, the efficacy and tolerability profile of olanzapine LAI is similar to oral olanzapine.

\section{Additional file}

Additional file 1: Mean changes in PANSS total scores during six weeks of treatment in olanzapine long-acting injection and oral olanzapine studies of acute schizophrenia. This file contains a figure depicting the visitwise mean changes in PANSS total scores for the 3 studies.

\section{Abbreviations}

BPRS: Brief Psychiatric Rating Scale; DSM: Diagnostic and Statistical Manual of Mental Disorders; LAI: long-acting injection; OLZ: Olanzapine; PANSS: Positive and Negative Syndrome Scale total score; PDSS: Post-injection Delirium/ Sedation Syndrome.
}

\section{Competing interests}

Holland C. Detke, Fangyi Zhao, and Michael M. Witte are full-time employees of Eli Lilly and Company.

\section{Acknowledgements}

Funding for this project was provided by Eli Lilly and Company (Indianapolis, Indiana, USA). The clinical trials included in the analyses were parts of larger clinical registration programs by Eli Lilly and Company. Eli Lilly contracted the editing of this manuscript with i3 Statprobe, a division of Ingenix, which is a subsidiary of UnitedHealth Group.

\section{Author details}

${ }^{1}$ Eli Lilly and Company, Indianapolis, IN, USA. ${ }^{2}$ Lilly USA, LLC, Indianapolis, IN USA.

\section{Authors' contributions}

HCD and FZ conceived and designed the analyses; FZ was responsible for statistics. HCD, FZ, and MMW contributed to the interpretation of results and drafting of the manuscript, and all reviewed and approved the final version of the manuscript.

Received: 24 October 2011 Accepted: 30 May 2012

Published: 30 May 2012

\section{References}

1. Komossa K, Rummel-Kluge C, Hunger H, Schmid F, Schwarz S, Duggan L, Kissling W, Leucht S: Olanzapine versus other atypical antipsychotics for schizophrenia. Cochrane Database Syst Rev 2010, 3:CD006654.

2. Ascher-Svanum H, Peng X, Faries D, Montgomery W, Haddad PM: Treatment patterns and clinical characteristics prior to initiating depot typical antipsychotics for nonadherent schizophrenia patients. BMC Psychiatry 2009, 9:46.

3. Glazer WM: The depot paradox. Behav Healthc 2007, 27:44-46.

4. Heres S, Hamann J, Kissling W, Leucht S: Attitudes of psychiatrists toward antipsychotic depot medication. J Clin Psychiatry 2006, 67:1948-1953.

5. Burns T: Knowledge about antipsychotic long-acting injections: bridging that gap. Br J Psychiatry Supp/ 2009, 52:S5-S6.

6. Llorca PM: Partial compliance in schizophrenia and the impact on patient outcomes. Psychiatry Res 2008, 161:235-247.

7. Weiden PJ, Kozma C, Grogg A, Locklear J: Partial compliance and risk of rehospitalization among California Medicaid patients with schizophrenia. Psychiatr Serv 2004, 55:886-891.

8. West JC, Wilk JE, Olfson M, Rae DS, Marcus S, Narrow WE, Pincus HA, Regier DA: Patterns and quality of treatment for patients with schizophrenia in routine psychiatric practice. Psychiatr Serv 2005, 56:283-291.

9. Lauriello J, Lambert T, Andersen S, Lin D, Taylor CC, McDonnell D: An 8week, double-blind, randomized, placebo-controlled study of olanzapine long-acting injection in acutely ill patients with schizophrenia. J Clin Psychiatry 2008, 69:790-799.

10. Kane JM, Detke HC, Naber D, Sethuraman G, Lin DY, Bergstrom RF, McDonnell D: Olanzapine long-acting injection: a 24-week, randomized, double-blind trial of maintenance treatment in patients with schizophrenia. Am J Psychiatry 2010, 167:181-189.

11. Frampton JE: Olanzapine long-acting injection: a review of its use in the treatment of schizophrenia. Drugs 2010, 70:2289-2313.

12. Lindenmayer J: Long-acting injectable antipsychotics: focus on olanzapine pamoate. Neuropsychiatr Dis Treat 2010, 6:261-267.

13. Naber D: Olanzapine pamoate for the treatment of schizophrenia. Expert Opin Pharmacother 2011, 12:627-633.

14. Beasley CM Jr, Tollefson G, Tran P, Satterlee W, Sanger T, Hamilton S: Olanzapine versus placebo and haloperidol: acute phase results of the North American double-blind olanzapine trial. Neuropsychopharmacology 1996, 14:111-123.

15. Beasley CM Jr, Hamilton SH, Crawford AM, Dellva MA, Tollefson GD, Tran PV, Blin O, Beuzen JN: Olanzapine versus haloperidol: acute phase results of the international double-blind olanzapine trial. Eur Neuropsychopharmacol 1997, 7:125-137.

16. Beasley CM Jr, Sanger T, Satterlee W, Tollefson G, Tran P, Hamilton S: Olanzapine versus placebo: results of a double-blind, fixed-dose olanzapine trial. Psychopharmacology (Berl) 1996, 124:159-167.

17. Simpson GM, Angus JWS: A rating scale for extrapyramidal side effects. Acta Psychiatr Scan Suppl 1970, 212:11-19.

18. Barnes TR: A rating scale for drug induced akathisia. Br J Psychiatry 1989, 154:672-676.

19. Kazis LE, Anderson JJ, Meenan RF: Effect sizes for interpreting changes in health status. Med Care 1989, 27(3 Suppl):S178-S189.

20. McDonnell DP, Detke HC, Bergstrom RF, Kothare P, Johnson J, Stickelmeye M, Sanchez-Felix MV, Sorsaburu S, Mitchell MI: Post-injection delirium/ sedation syndrome in patients with schizophrenia treated with 
olanzapine long-acting injection. II: investigations of mechanism. $B M C$ Psychiatry 2010, 10:45.

21. McDonnell DP, Andersen SW, Detke HC, Zhao F, Watson SB: Long-term safety and tolerability of open-label olanzapine long-acting injection in the treatment of schizophrenia: 190-week interim results. Clinical Medicine Insights: Psychiatry 2011, 3:37-47.

22. Citrome L, Holt RI, Walker DJ, Hoffman VP: Weight gain and changes in metabolic variables following olanzapine treatment in schizophrenia and bipolar disorder. Clin Drug Investig 2011, 31:455-482.

23. Detke HC, McDonnell DP, Brunner E, Zhao F, Sorsaburu S, Stefaniak VJ, Corya SA: Post-injection delirium/sedation syndrome in patients with schizophrenia treated with olanzapine long-acting injection. I: analysis of cases. BMC Psychiatry 2010, 10:43.

24. Kane JM, Leucht $\mathrm{S}$ : Unanswered questions in schizophrenia clinical trials. Schizophr Bull 2008, 34:302-309.

25. Masand PS, Roca M, Turner MS, Kane JM: Partial adherence to antipsychotic medication impacts the course of illness in patients with schizophrenia: a review. Prim Care Companion J Clin Psychiatry 2009, 11:147-154.

26. Valenstein M, Ganoczy D, McCarthy JF, Myra KH, Lee TA, Blow FC: Antipsychotic adherence over time among patients receiving treatment for schizophrenia: a retrospective review. J Clin Psychiatry 2006, 67:1542-1550.

27. Detke HC, Zhao F, Garhyan P, Carslon J, McDonnell D: Dose correspondence between olanzapine long-acting injection and oral olanzapine: reccomendations for switching. Int Clin Psychopharmaco 2011, 26:35-42.

doi:10.1186/1471-244X-12-51

Cite this article as: Detke et al:: Efficacy of olanzapine long-acting

injection in patients with acutely exacerbated schizophrenia: an insight from effect size comparison with historical oral data. BMC Psychiatry 2012 12:51.

\section{Submit your next manuscript to BioMed Central and take full advantage of:}

- Convenient online submission

- Thorough peer review

- No space constraints or color figure charges

- Immediate publication on acceptance

- Inclusion in PubMed, CAS, Scopus and Google Scholar

- Research which is freely available for redistribution 